\title{
OPEN Fall-related functional impairments in patients with neurological gait disorder
}

\author{
Angela Ehrhardt ${ }^{1 凶}$, Pascal Hostettler ${ }^{1}$, Lucas Widmer ${ }^{1}$, Katja Reuter ${ }^{1}$, \\ Jens Alexander Petersen ${ }^{1}$, Dominik Straumann ${ }^{1}$ \& Linard Filli ${ }^{1,2,3}$
}

Falls are common in patients with neurological disorders and are a primary cause of injuries. Nonetheless, fall-associated gait characteristics are poorly understood in these patients. Objective, quantitative gait analysis is an important tool to identify the principal fall-related motor characteristics and to advance fall prevention in patients with neurological disorders. Fall incidence was assessed in 60 subjects with different neurological disorders. Patients underwent a comprehensive set of functional assessments including instrumented gait analysis, computerized postural assessments and clinical walking tests. Determinants of falls were assessed by binary logistic regression analysis and receiver operator characteristics (ROC). The best single determinant of fallers was a step length reduction at slow walking speed reaching an accuracy of $67.2 \%$ (ROC AUC: $0.669 ; p=0.027$ ). The combination of 4 spatio-temporal gait parameters including step length and parameters of variability and asymmetry were able to classify fallers and non-fallers with an accuracy of $81.0 \%$ (ROC AUC: $0.882 ; p<0.001$ ). These findings suggest significant differences in specific spatio-temporal gait parameters between fallers and non-fallers among neurological patients. Fall-related impairments were mainly identified for spatio-temporal gait characteristics, suggesting that instrumented, objective gait analysis is an important tool to estimate patients' fall risk. Our results highlight pivotal fall-related walking deficits that might be targeted by future rehabilitative interventions that aim at attenuating falls.

Falls are common in elderly people and are often associated with serious injuries, reduced mobility and loss of independency ${ }^{1} .25-35 \%$ of people older than 65 years fall regularly and more than half of falls in these patients occur during walking ${ }^{2}$. There is a multitude of studies investigating functional determinants or predictors of falls in healthy elderly subjects. Identified functional impairments that differentiate healthy fallers from non-fallers are heterogeneous and depend on the functional outcomes that are assessed in the particular studies ${ }^{3}$. Most common techniques assessing fall risk comprise motor performance tests, questionnaires, and laboratory-based, instrumented measurements (e.g. force platforms, computerized walkways, accelerometers etc.). Spatio-temporal gait parameters, in particular reduced step length and increased variability of stance and stride time, were shown to be valid determinants of falls in elderly subjects ${ }^{2-13}$. In addition, computerized outcomes of postural stability (e.g. sway assessment) and clinical walking outcomes (e.g. timed-up and go (TUG), functional gait assessment (FGA)) revealed significant correlations with fall risk in elderly people ${ }^{14-17}$.

In contrast to healthy subjects, potential predictors of falls are poorly investigated in patients with neurological disorders. Fall incidence is reported 2-4 times higher in patients with neurological disorders than in healthy subjects of similar age $\mathrm{e}^{18,19}$ and $46 \%$ of the neurological patients reveal one or more falls per year ${ }^{20}$. There are studies examining fall-associated functional impairments regarding clinical assessments ${ }^{21,22}$, postural outcomes ${ }^{23,24}$ and computerized gait analysis ${ }^{25-28}$. There is, however, only sparse knowledge on a valid, standardized determinant of falls that might apply to different neurological gait pathologies. Moreover, there are virtually no studies performing a comprehensive functional test battery including manifold biomechanical parameters of stance and gait, as well as of standardized clinical walking tests to screen for predominant functional impairments that are characteristic of fallers with neurological movement disorders.

The aim of this study was to identify functional impairments regarding posture and gait that are most characteristic of fallers in patients with neurological gait disorders. In contrast to earlier studies, we examined

${ }^{1}$ Department of Neurology, University Hospital and University of Zurich, Zurich, Switzerland. ${ }^{2}$ Spinal Cord Injury Center, Balgrist University Hospital, Zurich, Switzerland. ${ }^{3}$ Swiss Center for Clinical Movement Analysis (SCMA), Balgrist Campus AG, Zurich, Switzerland. ${ }^{\circledR}$ email: Angela.ehrhardt@usz.ch 


\section{Inclusion criteria}

- Signed informed consen

- Completed fall questionnaire

- Complete dataset of clinical walking tests (timed 25-foot walk

6-min walk test, timed up and go, functional gait assessment)

- Complete set of postural assessments (body sway during normal

standing, Romberg position with eyes open, Romberg position with eyes closed)

- Complete dataset of treadmill walking without handrail support at 1,2 and $3 \mathrm{~km} / \mathrm{h}$

- Patients $\geq 18$ years with a defined neurological diagnosis
Exclusion criteria

- Lack of informed consent or fall questionnaire

- Incomplete dataset of clinical walking tests, postural and $\bullet$ locomo-

tor assessments

- Patients that were not able to understand the content of the fall

questionnaire

- Participants with suspected psychogenic gait disorders or major cognitive deficits

- Major orthopedic, cardiovascular or pulmonary disorders affecting walking function

- Patients with a secondary neurological diagnosis

Table 1. In- and exclusion criteria.

\begin{tabular}{|c|c|}
\hline & Patients \\
\hline Number of patients & 58 \\
\hline Age (years), mean \pm SD & $52.5 \pm 14.5$ \\
\hline Gender, proportion of female & $25 / 58$ \\
\hline Fallers, proportion of fallers & $29 / 58$ \\
\hline \multicolumn{2}{|l|}{ Neurological condition, number of patients } \\
\hline $\begin{array}{l}\text { Inflammatory CNS diseases (MS: } \mathrm{n}=37 ; \mathrm{CIS}: \mathrm{n}=2 \text { ) } \\
\text { Peripheral neuropathies } \\
\text { Vertigo syndromes } \\
\text { Cerebrovascular CNS diseases } \\
\text { Myopathies } \\
\text { Idiopathic normal pressure hydrocephalus }\end{array}$ & $\begin{array}{l}39 \\
7 \\
5 \\
3 \\
2 \\
2\end{array}$ \\
\hline
\end{tabular}

Table 2. Demographic and clinical characteristics of the study population. CNS central nervous system, $S D$ standard deviation, $M S$ multiple sclerosis, CIS clinically isolated syndrome.

determinants of falls in a heterogeneous group of neurological patients comprising, among others, patients with multiple sclerosis, polyneuropathy, vertigo syndromes, cerebrovascular diseases and idiopathic normal pressure hydrocephalus. Given the heterogeneous literature on determinants of falls, we screened a large, multimodal set of different postural and clinical outcome measures as well as gait parameters at different walking speeds for their ability to differentiate fallers from non-fallers. Gait assessments at differential walking speeds were performed to place varying demands on locomotor function (e.g. slow walking speeds demand for high dynamic stability, faster speeds require enhanced force and coordinative abilities ${ }^{29}$ ). Our findings might help to improve upon the understanding of falls in neurological patients and thereby promote the designing of therapeutic strategies that aim at preventing or reducing the incidence of falls in neurological patients.

\section{Methods}

Participants. Functional data was retrospectively analyzed from participants that were routinely examined at the Locomotion Research Laboratory of the Department of Neurology at the University Hospital Zurich. There was no specific recruitment of patients for this study. All participants provided written informed consent regarding a further use of their clinical data for the purpose of clinical research. All measurements were performed in accordance with the Declaration of Helsinki. The procedures were approved by the Zurich cantonal ethics committee (project ID: 2017-01459). Patients with a defined neurological diagnosis and no major orthopedic, cardiovascular or pulmonary disorders affecting walking function were included (Tables 1,2 ). For inclusion, patients needed to be able to complete all clinical walking tests, the postural assessments and the kinetic walking trials. All patients were able to understand the content of the fall questionnaire. Participants with suspected psychogenic gait disorders or major cognitive deficits were excluded from the analysis. Patients who were not able to walk without holding onto the handrails of the treadmill at 1,2 and $3 \mathrm{~km} / \mathrm{h}$ where excluded: Furthermore, we excluded groups with only one participant (Fig. 1, Table 1).

\section{Experimental procedures}

Participants were asked to complete a questionnaire assessing the incidence of falls within 6 months prior to data analysis. Considering the potential for recall bias the period of retrospective fall assessment was limited to six months. A fall was defined as an unexpected event where a patient lost his balance and reached a position on the floor ${ }^{9,11,18}$. Collapses because of internistic events such as seizures, ischemic attacks or syncopes were not defined as falls. Participants who had at least one fall within the last 6 months where classified as "fallers".

All participants underwent a comprehensive gait analysis and performed different posturographic measurements. Clinical gait tests consisted of the timed 25 -foot walk (T25FW) ${ }^{30}$ assessing maximal walking speed, the 6-min walk test $(6 \mathrm{MWT})^{31}$ measuring short-time endurance, the timed up and go test (TUG) ${ }^{32}$ assessing 


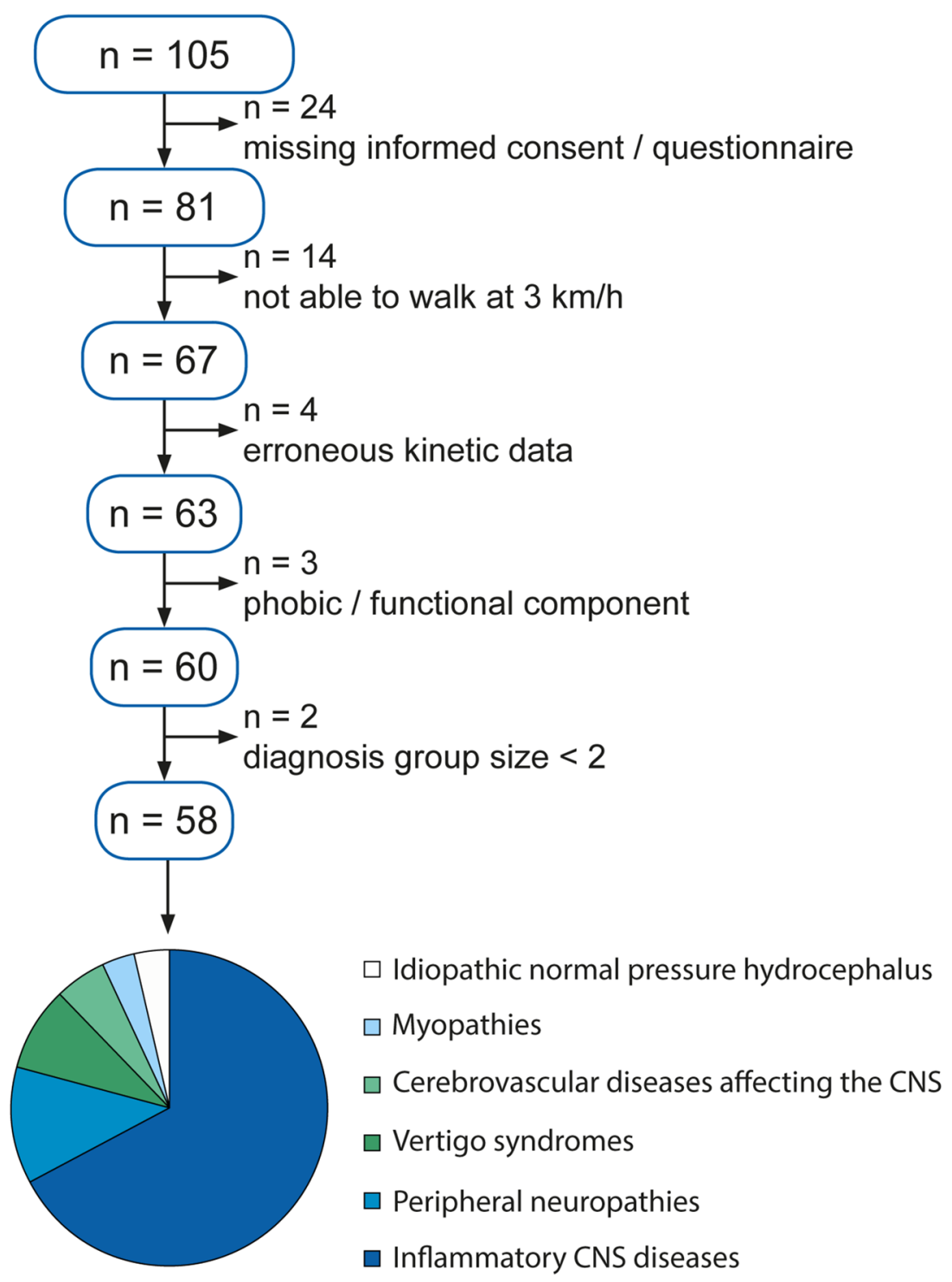

Figure 1. Flowchart of the sampling procedure.

functional mobility and daily locomotor function as well as the functional gait assessment (FGA) $)^{33}$ measuring dynamic stability and balance.

Instrumented, treadmill-based gait analysis was used to objectively assess patients' walking patterns (i.e. quality of walking) at 1,2 and $3 \mathrm{~km} / \mathrm{h}$. Different gait speeds were used to assess distinct sensorimotor abilities (e.g. high stability requirements at slow speed vs. enhanced force and coordinative requirements at faster speeds). Comprehensive gait profiles consisting of 20 kinetic gait parameters were produced for each patient at each gait velocity. These walking parameters served to assess different aspects of locomotion such as step length, gait phases, dynamic instability, asymmetry and gait variability, thus being able to comprehensively characterize patients' walking pathologies (Table 3). All participants walked barefoot for at least $30 \mathrm{~s}$ per trial on an instrumented treadmill (120 Hz, FDM-T, Zebris Medical GmbH, Germany) without holding onto the handrails. Prior to data recording, all participants were familiarized to treadmill walking for at least $7 \mathrm{~min}$ at each gait speed $(1,2$ and $3 \mathrm{~km} / \mathrm{h}$ ) to prevent major adaptations of the gait pattern and to reduce the fear of falling ${ }^{34}$. After this initial familiarization period, kinetic gait data was recorded over $30 \mathrm{~s}$ at each velocity (1, 2 and $3 \mathrm{~km} / \mathrm{h}$ ). Participants were instructed not to talk or gesticulate and to naturally move/swing their arms. Moreover, patients were asked to fix their gaze onto a cross projected on a screen (22" LCD monitor) positioned at eye height in front of the treadmill at any time during the recordings ${ }^{35}$. After the training and between the trials patients were allowed to have a short break if required.

Postural stability was quantified by center of pressure $(\mathrm{CoP})$ sway measurements during normal standing (20 cm distance between left and right hallux) and during the Romberg test (i.e. feet close together) with eyes open and closed ${ }^{36}$. Postural assessments were performed on the instrumented treadmill. Participants performed four trials of $15 \mathrm{~s}$ for each postural condition. CoP sway velocity $(\mathrm{mm} / \mathrm{s})$ and the $95 \%$ confidence interval of the 


\begin{tabular}{|c|c|c|}
\hline Spatio-temporal kinetic gait parameters & & \\
\hline Locomotor domain & Gait parameters & Units \\
\hline \multirow[t]{2}{*}{ Limb excursion } & Step length (left, right) & $\mathrm{mm}$ \\
\hline & Step time (left, right) & $\mathrm{ms}$ \\
\hline \multirow[t]{3}{*}{ Gait phases } & Stance phase (left/right) & $\%$ \\
\hline & Swing phase (left/right) & $\%$ \\
\hline & Double-limb support & $\%$ \\
\hline Stability & Step width & $\mathrm{mm}$ \\
\hline \multirow[t]{3}{*}{ Asymmetry } & Step length asymmetry & $\%$ \\
\hline & Step time asymmetry & $\%$ \\
\hline & Swing phase asymmetry & $\%$ \\
\hline \multirow[t]{5}{*}{ Variability } & COV step length (left, right) & $\%$ \\
\hline & COV step time (left, right) & $\%$ \\
\hline & COV step width & $\%$ \\
\hline & CoP variability ant-post & $\mathrm{mm}$ \\
\hline & CoP variability med-lat & $\mathrm{mm}$ \\
\hline \multicolumn{3}{|l|}{ Clinical walking tests } \\
\hline Test & Readouts & Units \\
\hline Timed 25-foot walk (T25FW) & Maximal walking speed & $\mathrm{s}$ \\
\hline 6-min walk test (6MWT) & Short-term endurance & $\mathrm{m}$ \\
\hline Timed-up and go (TUG) & Mobility, daily walking function & s \\
\hline Functional gait assessment (FGA) & Dynamic stability, balance & Points \\
\hline \multicolumn{3}{|l|}{ Postural stability measures } \\
\hline Test & Stability measures & Units \\
\hline \multirow{2}{*}{ Normal stance (feet $20 \mathrm{~cm}$ apart) } & Sway velocity & $\mathrm{mm} / \mathrm{s}$ \\
\hline & Sway area (95\% confidence) & $\mathrm{mm}^{2}$ \\
\hline \multirow{2}{*}{ Romberg stance (eyes open) } & Sway velocity & $\mathrm{mm} / \mathrm{s}$ \\
\hline & Sway area (95\% confidence) & $\mathrm{mm}^{2}$ \\
\hline \multirow{2}{*}{ Romberg stance (eyes closed) } & Sway velocity & $\mathrm{mm} / \mathrm{s}$ \\
\hline & Sway area (95\% confidence) & $\mathrm{mm}^{2}$ \\
\hline
\end{tabular}

Table 3. Gait and postural assessments performed in this study. Walking parameters indicative of various locomotor aspects (e.g. stability, asymmetry, variability) were assessed at gait speeds of $1 \mathrm{~km} / \mathrm{h}, 2 \mathrm{~km} / \mathrm{h}$ and $3 \mathrm{~km} / \mathrm{h}$ (subtable on the top). Four clinical walking tests were assessed to quantify patients' walking performance (intermediate table). Three different postural test conditions were performed to measure patients' postural stability (table at the bottom). CoP center of pressure, $C O V$ coefficient of variance, $m m$ millimeter, min minute, $m s$ milliseconds.

ellipse sway area $\left(\mathrm{mm}^{2}\right)$ were used as outcome parameters of postural stability. For each test condition, the trial with the lowest sway velocity (i.e. the most stable trial) was selected for analysis.

Kinetic data of the gait and posture trials were acquired by a pressure plate that was integrated in the treadmill (DMTHM-M-2i System, Zebris Medical GmbH, Germany). Parameter raw data per step cycle and patient was extracted from the Zebris FDM software and further processed in Maltab using customized scripts (Matlab 2017b, Mathworks Inc., Natick, USA).

Data analysis. Statistical analysis was performed with SPSS statistical software (V23.0; IBM Corp., Armonk, NY). Group differences in kinetic gait patterns, clinical walking performance and postural stability between fallers and non-fallers were assessed by 2-way ANOVA with the factors fallers and parameters. Post-hoc corrections for multiple testing (Sidak's correction) was performed to assess group differences on the level of single parameters. Binary logistic regression (Wald forward stepwise) was used to identify determinants of falls and to determine their contribution to separate fallers from non-fallers ${ }^{13}$. Results of the kinetic gait analysis, the clinical gait tests and the posturographic assessments (i.e. 70 factors) were included in the binary logistic regression. The dependent variable was defined as fall status, whereby fallers were defined as patients experiencing at least one fall within the last 6 months prior to the clinical examination. Stepwise forward regressions were set at $p>0.05$ for entry and removal of a variable. All analyses have been adjusted for the covariates age and gender.

Fall-related functional parameters as defined by binary logistic regression analysis were further assessed by receiver operator characteristics (ROC) with bootstrapping to obtain $95 \%$ confidence intervals. ROC analysis was performed for the strongest single determinant of falls, as well as for the combinations of fall determinants. 


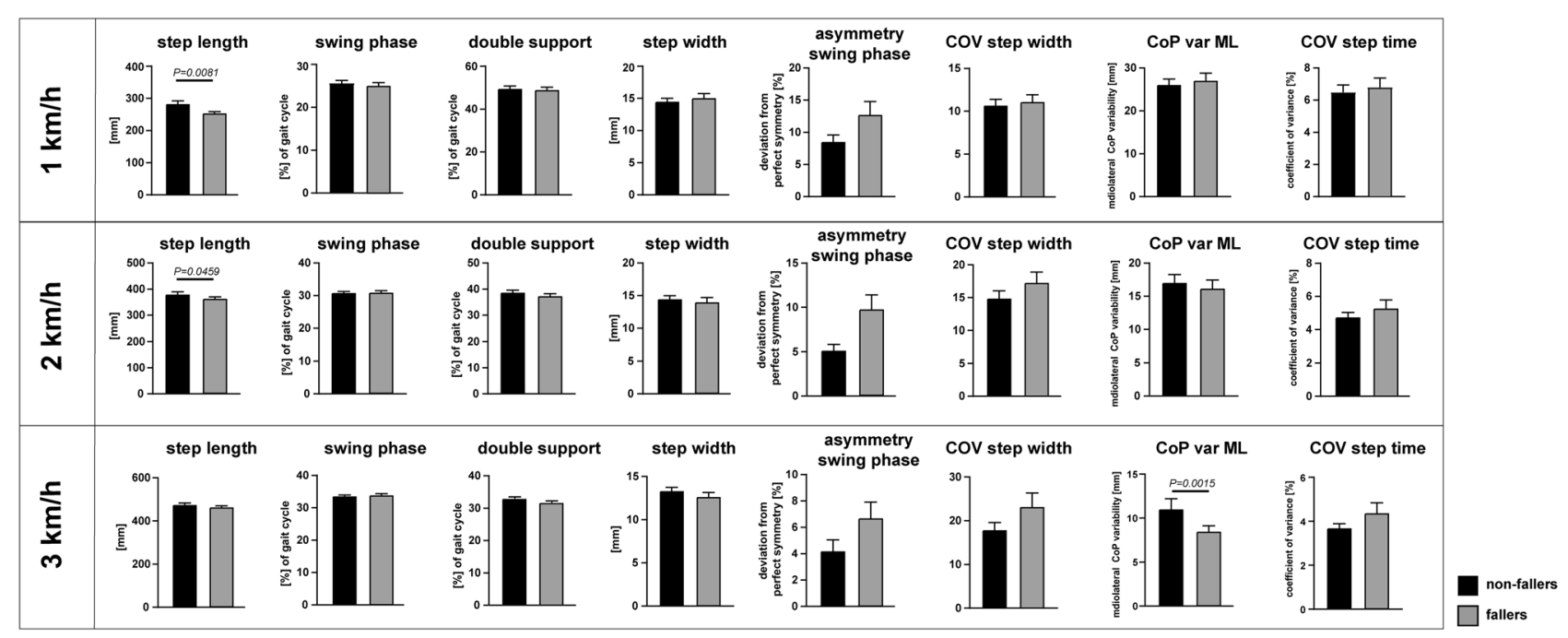

Figure 2. Key spatio-temporal gait parameters at different walking speeds in fallers vs. non-fallers. Spatiotemporal gait parameters were compared between fallers vs. non-fallers of our cohort. Significant differences (indicated by P-values above parameters) are based on 2-way ANOVA followed by Sidak?s post hoc correlation for all 20 walking parameters at the respective gait speeds of $1 \mathrm{~km} / \mathrm{h}, 2 \mathrm{~km} / \mathrm{h}$ and $3 \mathrm{~km} / \mathrm{h}$. CoP center of pressure, $\mathrm{COV}$ coefficient of variance, var $M L$ variability in mediolateral direction.

\section{Results}

Fifty-eight patients ( 25 females, age: $52.5 \pm 14.5$ years) with gait disorders due to different neurological conditions were analyzed (Table 2). Five patients with suspected psychogenic origin of gait disorders were excluded from the analysis. Twenty-nine of 58 patients reported falls within the last 6 months prior to the functional assessments. None of the patients used assistive walking aids during the clinical walking tests or the instrumented gait analysis.

Characterizing gait and postural deviations in fallers vs. non-fallers. In a first step, we analyzed group differences in fallers vs. non-fallers regarding kinetic gait patterns, clinical walking performance and postural stability. There were no significant differences between fallers vs. non-fallers regarding parameters of postural stability (2-way ANOVA, factors "fallers x parameters"; $\mathrm{P}=0.2611$ ) and clinical walking performance (2-way ANOVA, $\mathrm{P}=0.1369)$. Spatio-temporal gait parameters over all three assessed gait velocities $(1,2$ and $3 \mathrm{~km} / \mathrm{h}$ ) also revealed no significant group differences (2-way ANOVA, $\mathrm{P}=0.1715$ ). Interestingly, the analysis of spatio-temporal gait parameters at individual gait velocities showed no group differences at slow walking speed (i.e. $1 \mathrm{~km} / \mathrm{h}$; 2 -way ANOVA; $\mathrm{P}=0.7580)$, but enhanced differences at higher gait velocities $(2 \mathrm{~km} / \mathrm{h} ; \mathrm{P}=0.1726)$ which reached statistical significance at $3 \mathrm{~km} / \mathrm{h}$ (2-way ANOVA, factor fallers; $\mathrm{P}=0.0178$; Fig. 2). At 1 and $2 \mathrm{~km} / \mathrm{h}$, reduction of step length was the only significant difference in fallers vs. non-fallers surviving the correction for multiple comparisons $(\mathrm{P}=0.0081$ and $\mathrm{P}=0.0459$ respectively; Sidak's correction $)$. At $3 \mathrm{~km} / \mathrm{h}$, mediolateral variability of the center of pressure was significantly reduced in fallers vs. non-fallers $(\mathrm{P}=0.0015 ;$ Fig. 2$)$.

Determinants of fallers vs. non-fallers. After characterizing gait and postural differences in fallers vs. non-fallers, we screened for the most potent determinants of falls. A total of 70 independent variables (Table 3; 20 gait parameters at 3 different gait velocities, 6 posturographic parameters, 4 clinical walking tests) were analyzed by a binary logistic regression analysis with the dependent variable defined as faller status (Table 4). The covariates age $(\mathrm{P}=0.417)$ and gender $(\mathrm{P}=0.185)$ did not have an influence on the analysis. The best single variable discriminating fallers vs. non-fallers was step length (right leg) at $1 \mathrm{~km} / \mathrm{h}$ reaching an accuracy of $67.2 \%$ (Nagelkerke coefficient of determination $\mathrm{R}^{2}=0.111$; specificity: $55.2 \%$; sensitivity: $79.3 \%$ ). Combining the three gait parameters (1) step length at $1 \mathrm{~km} / \mathrm{h},(2) \mathrm{CoP}$ variability mediolateral at $3 \mathrm{~km} / \mathrm{h}$ and (3) asymmetry of swing phase at $2 \mathrm{~km} / \mathrm{h}$ resulted in an accuracy of $72.4 \%$ (Nagelkerke coefficient of determination $\mathrm{R}^{2}=0.486$; specificity: $72.4 \%$; sensitivity: $72.4 \%$ ) to classify fallers and non-fallers. The best accuracy in discriminating fallers vs. non-fallers (accuracy: $81.0 \%$; specificity: $79.3 \%$; sensitivity: $82.8 \%$; Nagelkerke coefficient of determination $\left.\mathrm{R}^{2}=0.558\right)$ was achieved using the combination of four kinetic factors: (1) step length at $1 \mathrm{~km} / \mathrm{h}$, (2) CoP variability mediolateral at $3 \mathrm{~km} / \mathrm{h}$, (3) asymmetry of swing phase at $2 \mathrm{~km} / \mathrm{h}$ and (4) COV (coefficient of variance) of step width at $3 \mathrm{~km} / \mathrm{h}$. The model using one determinant only (model A: step length at $1 \mathrm{~km} / \mathrm{h}$ ) revealed the highest sensitivity (i.e. correctly assigning fallers), however, the lowest specificity (i.e. correctly assigning non-fallers) of all logistic regression models. Posturographic parameters or clinical walking outcomes were not sufficiently powerful to improve upon the classification of fallers and non-fallers in our population.

ROC curve analysis of principal determinants of fallers. Detailed sensitivity and specificity characteristics of the strongest fall-related determinants (as defined by binary logistic regression analysis) were further assessed by receiver operator characteristics (ROC; Fig. 3). The best single parameter (i.e. right step length at 


\begin{tabular}{|c|c|c|c|}
\hline \multirow[b]{2}{*}{ Observed } & \multicolumn{3}{|l|}{ Predicted } \\
\hline & Non-fallers & Fallers & Percentage correct \\
\hline \multicolumn{4}{|c|}{ A: Model comprises step length at $1 \mathrm{~km} / \mathrm{h}$} \\
\hline Non-fallers & 17 & 13 & 56.7 \\
\hline \multirow[t]{2}{*}{ Fallers } & 5 & 25 & 83.3 \\
\hline & & & 70.0 \\
\hline \multicolumn{4}{|c|}{ B: Model comprises A \& CoP variability med-lat at $3 \mathrm{~km} / \mathrm{h}$} \\
\hline Non-fallers & 20 & 10 & 66.7 \\
\hline \multirow[t]{2}{*}{ Fallers } & 8 & 22 & 73.3 \\
\hline & & & 70.0 \\
\hline \multicolumn{4}{|c|}{ C: Model comprises B \& asymmetry swing phase at $2 \mathrm{~km} / \mathrm{h}$} \\
\hline Non-fallers & 22 & 8 & 73.3 \\
\hline \multirow[t]{2}{*}{ Fallers } & 8 & 22 & 73.3 \\
\hline & & & 73.3 \\
\hline \multicolumn{4}{|c|}{ D: Model comprises C \& COV step width at $3 \mathrm{~km} / \mathrm{h}$} \\
\hline Non-fallers & 24 & 6 & 80.0 \\
\hline \multirow[t]{2}{*}{ Fallers } & 5 & 25 & 83.3 \\
\hline & & & 81.7 \\
\hline
\end{tabular}

Table 4. Prediction of fallers vs. non-fallers. Classification tables illustrating observed and predicted outcomes of the binary logistic regression model using (A) the single most predictive factor (i.e. step length (right leg) at $1 \mathrm{~km} / \mathrm{h}$ ), as well as combinations of the most predictive factors (B-D) for falls. CoP center of pressure, $C O V$ coefficient of variance, med-lat mediolateral.

ROC Curve: Model A

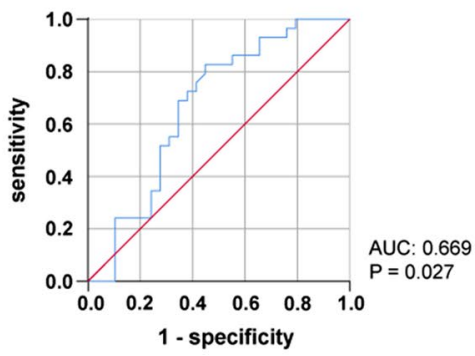

ROC Curve: Model C

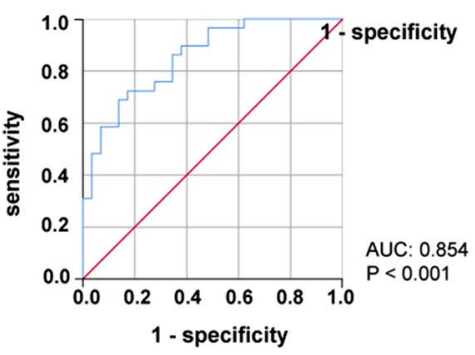

ROC Curve: Model B

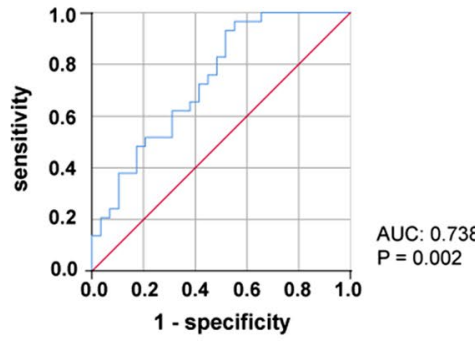

Model A: $\quad$ step length (right leg) at $1 \mathrm{~km} / \mathrm{h}$

Model B:

step length (right leg) at $1 \mathrm{~km} / \mathrm{h}$ CoP variability med-lat at $3 \mathrm{~km} / \mathrm{h}$

Model C:

step length (right leg) at $1 \mathrm{~km} / \mathrm{h}$ CoP variability med-lat at $3 \mathrm{~km} / \mathrm{h}$ asymmetry of swing phase at $2 \mathrm{~km} / \mathrm{h}$

Model D: step length (right leg) at $1 \mathrm{~km} / \mathrm{h}$ CoP variability med-lat at $3 \mathrm{~km} / \mathrm{h}$ asymmetry of swing phase at $2 \mathrm{~km} / \mathrm{h}$ COV step width at $3 \mathrm{~km} / \mathrm{h}$

Figure 3. Receiver operator characteristics curves of the best predictors of falls. The best predictors of falls were further analyzed by receiver operator characteristics (ROC) curves to investigate the specificity and sensitivity of these variables. ROC curves were assessed for the best single predictor (Model A; top left), as well as for the best combinations of predictors (Model B-D). AUC and P-values of the ROC curves are highlighted at the right bottom corner of each panel. $A U C$ area under the curve, $C o P$ center of pressure, $C O V$ coefficient of variance.

$1 \mathrm{~km} / \mathrm{h}$ ) reached a ROC area under the curve (AUC) of 0.669 suggesting valid but rather modest ability to correctly classify fallers and non-fallers. The combination of different fall-related determinants reached AUC values of $0.738,0.854$ and 0.882 for combinations of 2,3 and 4 variables, respectively (Fig. 3 ). These combinations thus reveal fair to good ability to distinguish between fallers and non-fallers. 
Fall-related functional characteristics in subjects with inflammatory CNS diseases. Analysis of functional determinants of falls in patients with inflammatory CNS diseases (i.e. multiple sclerosis $(n=37)$, clinically isolated syndrome $(n=2)$ ) identified enhanced variability of step width at $3 \mathrm{~km} / \mathrm{h}$ as only factor associated with falls. The power of this factor is considered moderate to low (accuracy: $61.5 \%$; specificity: $77.3 \%$; sensitivity: 41.2\%; Nagelkerke coefficient of determination $\left.\mathrm{R}^{2}=0.151\right)$. ROC analysis revealed an AUC of $0.698(\mathrm{p}=0.08)$. The covariates age $(\mathrm{P}=0.828)$ and gender $(\mathrm{P}=0.140)$ did not have an influence on the analysis.

\section{Discussion}

This study aimed at determining the principal postural and gait impairments that are associated with falls in patients with various neurological disorders. A comprehensive set of kinetic gait parameters, standardized clinical walking tests and posturographic parameters was assessed to identify the main determinants of falls in our cohort of neurological patients. The strongest fall-related functional impairment was a reduction of right step length at slow walking speed. The gait phenotype that was most strongly associated with falls was characterized by reduced step length, restricted mediolateral CoP variability, enhanced swing phase asymmetry and increased variability of step width. Our results highlight the main biomechanical characteristics of fallers with neurological disorders. The identified parameters represent paramount markers of enhanced fall risk in various neurological gait disorders that should be considered in physiotherapeutical strategies that aim at preventing falls in neurological patients. Moreover, objective monitoring of these fall-related gait parameters might allow to optimize treatments for gait disorders (e.g. medications, physical therapy etc.) in the clinical setting.

The strongest spatio-temporal gait determinants of falls identified in this study (i.e. reduced step length) were previously described as predictors of falls in healthy elderly people $e^{4,6,12,13}$. However, in all of the mentioned studies fallers and non-fallers walked at their self-selected speeds, often resulting in slower walking speeds in fallers than non-fallers. As most gait parameters (including step length) show significant adaptations in response to changing gait speeds $s^{37,38}$, differential walking speeds between the investigated groups confound an accurate analysis of group characteristics. In the present study, we assessed kinetic walking parameters at three different, fixed walking speeds in all patients. This allowed us to perform precise inter-subject comparisons that are not confounded by varying gait speeds between participants. Analogous to our study, Barak et al. ${ }^{2}$ investigated stride length at fixed gait speeds and found shorter stride lengths in elderly fallers vs. non-fallers. Whereas there is robust evidence for step length reduction being a valid biomechanical determinant of falls in healthy elderly subjects, there is only sparse data on neurological patients. A recent study reported a significant reduction in step length in fallers vs. non-fallers in chronic stroke survivors ${ }^{27}$. Socie \& Sosnoff ${ }^{39}$ found a non-significant tendency towards shorter steps in people with multiple sclerosis (MS) and previous fall history. Step length reduction might reflect a compensatory strategy of neurological patients to counteract impairments in muscle strength and balance function ${ }^{40,41}$. Whether step length reduction is a primary fall-related gait characteristic or a secondary adaptation in response to another gait impairment remains to be clarified. The right-sided shortening of step length in our study might be explained by the fact that most patients show asymmetric gait deficits which-in our population-lead to an overall step length reduction that is more pronounced on the right side.

Reduced variability of the CoP in the mediolateral dimension was identified as important determinant of falls in our cohort. Whereas other parameters of gait variability are usually enhanced in fallers vs. non-fallers in healthy elderly fallers ${ }^{2,3,5-10,42}$, mediolateral variability of the COP was reduced in fallers vs. non fallers in our study. This finding conforms to a previous study that investigated mediolateral gait dynamics during walking in stroke survivors: the authors reported reduced mediolateral pelvis displacement in fallers vs. non-fallers with chronic stroke ${ }^{43}$. Reduced mediolateral variability might be an adaptation to stabilize the gait pattern which is also found in fall-prone populations other than stroke patients.

Asymmetric walking patterns are frequently observed in patients with neurological disorders and usually derive from unilaterally accentuated weakness, spasticity or sensory deficits ${ }^{44}$. We found that left-right asymmetry of swing phase is a biomechanical hallmark of fallers in patients with neurological disorders. Swing phase asymmetry has been reported previously in people with $\mathrm{MS}^{37,45,46}$ and other studies reported enhanced gait asymmetry in people with Parkinson's disease and stroke ${ }^{47-49}$. Kasser et al. ${ }^{45}$ conducted a logistic regression analysis to assess different balance, gait and strength parameters that are associated with falls in a group of female patients with MS. The authors identified stance time asymmetry as important determinant of falls in this population. Enhanced left-right asymmetry of gait parameters often results in claudication and reduced dynamic stability, thus likely increasing patients' fall risk.

Our results demonstrated increased step width variability in fallers vs. non-fallers. This is in agreement with previous studies on fall-related determinants in healthy elderly subjects ${ }^{3,7,12}$. Additionally, Rochester et al. ${ }^{50}$ reported increased step width variability in fallers with Parkinson's disease in comparison to healthy controls. The aforementioned studies underpin the value of this gait parameter to serve as biomechanical marker of gait instability and falls.

Enhanced variability of step width was identified as single fall-related determinant in a sub-analysis in patients with inflammatory CNS diseases (i.e. multiple sclerosis (MS; $n=37$ ), clinically isolated syndrome (CIS; $n=2)$ ). Whereas previous studies identified this gait parameter as important hallmark of MS-related gait deficits ${ }^{37,51,52}$ there are, to our knowledge, no findings that related enhanced step width variability with increased risk of falls in patients with MS or CIS.

Our data demonstrates accentuated gait pattern differences between fallers and non-fallers with increasing gait velocity. This indicates that faster walking speeds might be more sensitive to detect fall-related gait pathologies, which is in line with previous reports on elderly healthy subjects ${ }^{2,6}$ and patients with $\mathrm{MS}^{53}$. These findings highlight the importance to assess gait patterns at different speeds, also including non-comfortable, high speeds that challenge patients and facilitate the detection of subtle gait abnormalities ${ }^{29}$. 
In this study, we assessed a comprehensive set of spatio-temporal gait parameters, standardized clinical walking tests and posturographic outcomes in a mixed population of neurological patients and examined the ability of these parameters to differentiate fallers from non-fallers. The superior power of spatio-temporal gait parameters to separate fallers from non-fallers might be explained by the fact that most falls actually occur during walking ${ }^{54}$. Enhanced fall prediction by spatio-temporal gait parameters vs. clinical gait tests is in line with earlier reports $^{6,10,14,27,55,56}$ and might derive from enhanced sensitivity and objectivity of instrumented, technical outcome measures. Two studies that assessed computerized gait and balance measures, as well as clinical measures in patients with chronic stroke demonstrated that spatio-temporal gait parameters were better determinants of falls than posturographic and clinical parameters ${ }^{43,49}$. Summarized, these findings suggest that spatio-temporal gait parameters are a sensitive tool to assess fall-related functional impairments in neurological patients. The findings of our study do not allow to draw conclusion whether the identified functional determinants of falls are unique to neurological patients or whether they can also be found in other populations (e.g. elderly fallers).

Moreover, we used different fixed velocities 1,2 and $3 \mathrm{~km} / \mathrm{h}$ ). A complete analysis of gait patterns across different walking speeds allows to assess different modalities of gait function (e.g. balance at slow walking; strength and coordination at higher speeds) in all subjects ${ }^{29}$, which valorizes the quality of our multimodal gait analysis.

Another strength is the comprehensive collection of gait and stance tests including instrumented kinetic gait analysis, clinical gait tests and posturographic stance analysis. Thus, we could show the primary eligibility of kinetic parameters to test for faller-specific gait adaptations.

Furthermore, for two reasons we see the heterogeneity of the sample as a strength to find faller-related adaptations of gait. First, a certain variety of neurological gait patterns (faller and non-faller) is needed to be able to detect a potential dominant parameter which contributes to a history of falls. Second, as even in patients with the same neurological disease gait pattern vary widely, we wanted to find a measurement technique which is sufficiently sensitive to that. To enhance the knowledge and treatment of neurological gait disorders this is an essential predisposition.

A limitation of this study is that the instrumented gait assessment on the treadmill might not allow to entirely transfer the findings to over ground walking. However, many studies showed that the basic locomotor pattern during treadmill walking is highly similar to walking over normal ground in subjects that were sufficiently familiarized to treadmill walking ${ }^{34,57-60}$. A strength of treadmill-based gait analysis is that gait speed can be fixed, thus allowing for an accurate inter-subject comparison of gait parameters without confounding effects of differential walking speed between subjects ${ }^{37,38}$. Moreover, the treadmill allows to sample numerous, continous step cycles that enhances the power of gait analysis. A limitation of this study is the exclusion of subjects that were not able to walk unassisted at $3 \mathrm{~km} / \mathrm{h}$ on the treadmill likely caused a bias towards good walkers. The use of handrails, however, substantially influences subjects' walking patterns, thus confounding an accurate biomechanical gait analysis. Furthermore, our population revealed a high amount of people with MS, whereas some of the other neurological diagnoses were rather rare. Hence, our data might primarily reflect findings of people with MS and do less apply to other neurological cohorts. Another limitation is that due to the retrospective design of the study data on cognitive function is not available. Subjects with major cognitive deficits were excluded. However, we cannot exclude that mild cognitive deficits have influenced the fill out of the questionnaire and the performance of the walking tests. Furthermore, fear of falling was not tested but could have influenced the walking pattern during all tests. As this factor cannot be eliminated it would be helpful to evaluate if the faller-specific adaptations are also correlated to the magnitude of fear of falling. Future studies should include the testing of cognitive function and fear of falling to see their influence on faller-specific gait adaptations. Another important point is the retrospective design of the fall questionnaire which might lead to an underestimation of fall incidents as the patient might not remember fall events in the long past. In order to reduce the risk of recall bias we restricted on the retrospective fall assessment to six months.

\section{Conclusion}

Our findings highlight the principal functional impairments that are related to falls in a mixed population of neurological patients. The results suggest that multimodal gait analysis is superior to posturographic or clinical walking outcomes in differentiating fallers from non-fallers. Reduced step length at slow walking speed was the strongest single determinant of fallers, probably reflecting a compensation strategy for impaired muscle strength and balance function. Gait asymmetry and altered variability values of gait parameters were additional gait characteristics of fallers. Our findings emphasize the most prominent locomotor determinants of falls and thus present potential key targets for future interventions aiming at preventing falls in neurological patients.

Received: 21 April 2020; Accepted: 17 November 2020

Published online: 03 December 2020

\section{References}

1. Ayoung-Chee, P. et al. Long-term outcomes of ground-level falls in the elderly. J. Trauma Acute Care Surg. 76(2), 498-503 (2014).

2. Barak, Y., Wagenaar, R. C. \& Holt, K. G. Gait characteristics of elderly people with a history of falls: A dynamic approach. Phys. Ther. 86(11), 1501-1510 (2006).

3. Hamacher, D., Singh, N. B., Van Dieen, J. H., Heller, M. O. \& Taylor, W. R. Kinematic measures for assessing gait stability in elderly individuals: A systematic review. J. R. Soc. Interface 8(65), 1682-1698 (2011).

4. Mortaza, N., Abu Osman, N. A. \& Mehdikhani, N. Are the spatio-temporal parameters of gait capable of distinguishing a faller from a non-faller elderly?. Eur. J. Phys. Rehabil. Med. 50(6), 677-691 (2014).

5. Thaler-Kall, K. et al. Description of spatio-temporal gait parameters in elderly people and their association with history of falls: Results of the population-based cross-sectional KORA-Age study. BMC Geriatr. 15(1) (2015).

6. Newstead, A. H., Walden, J. G. \& Gitter, A. J. Gait variables differentiating fallers from nonfallers. J. Geriatr. Phys. Ther. 30(3), 93-101 (2007). 
7. Maki, B. E. Gait changes in older adults : Predictors of falls or indicators of fear ?. J. Am. Geriatr. Soc. 45(3), 1-12 (1997).

8. Van Schooten, K. S. et al. Daily-life gait quality as predictor of falls in older people: A 1-year prospective cohort study. PLoS ONE 11(7), 1-13 (2016).

9. Callisaya, M. L. et al. Gait, gait variability and the risk of multiple incident falls in older people: A population-based study. Age Ageing 40(4), 481-487 (2011).

10. Hausdorff, J. M., Rios, D. A. \& Edelberg, H. K. Gait variability and fall risk in community-living older adults: A 1-year prospective study. Arch. Phys. Med. Rehabil. 82(8), 1050-1056 (2001).

11. Verghese, J., Holtzer, R., Lipton, R. B. \& Wang, C. Quantitative gait markers and incident fall risk in older adults. J. Gerontol. Ser. A Biol. Sci. Med. Sci. 64(8), 896-901 (2009).

12. Brach, J. S., Berlin, J. E., Vanswearingen, J. M., Newman, A. B., Studenski, S. A. Too much or too little step width variability is associated with a fall history in older persons who walk at or near normal gait speed 8, 1-8 (2005).

13. König, N., Taylor, W. R., Armbrecht, G., Dietzel, R. \& Singh, N. B. Identification of functional parameters for the classification of older female fallers and prediction of 'first-time' fallers. J. R. Soc. Interface 11(97), 1 (2014).

14. Wrisley, D. M. \& Kumar, N. Functional gait assessment : Awelling older adults. Phys. Ther. 90(4), 1-13 (2010).

15. Melzer, I., Me, T., Di, B., Mcavay, G. Postural stability in the elderly : A comparison between fallers and non-fallers. $602-607$ (2003).

16. Johansson, J., Nordström, A., Gustafson, Y., Westling, G. \& Nordström, P. Increased postural sway during quiet stance as a risk factor for prospective falls in community-dwelling elderly individuals. Age Ageing 46(6), 964-970 (2017).

17. Howcroft, J., Lemaire, E. D., Kofman, J. \& McIlroy, W. E. Elderly fall risk prediction using static posturography. PLoS ONE 12(2), $1-13$ (2017).

18. Stolze, H. et al. Falls in frequent neurological diseases: Prevalence, risk factors and aetiology. J. Neurol. 251(1), 79-84 (2004).

19. Xu, T. et al. Risk factors for falls in community stroke survivors: A systematic review and meta-analysis. Arch. Phys. Med. Rehabil. 99(3), 563-573.e5 (2018).

20. Homann, B. et al. The impact of neurological disorders on the risk for falls in the community dwelling elderly: A case-controlled study. BMJ Open 3(11), 1-9 (2013).

21. Leddy, A. L., Crowner, B. E. \& Earhart, G. M. Functional gait assessment and balance evaluation system test: Reliability, validity, sensitivity, and specificity for identifying individuals with Parkinson disease who fall. Phys. Ther. 91(1), 102-113 (2011).

22. Vance, R. C., Healy, D. G., Galvin, R. \& French, H. P. Dual tasking with the timed 'up \& go' test improves detection of risk of falls in people with Parkinson disease. Phys. Ther. 95(1), 95-102 (2015).

23. Prosperini, L., Fortuna, D., Giannì, C., Leonardi, L. \& Pozzilli, C. The diagnostic accuracy of static posturography in predicting accidental falls in people with multiple sclerosis. Neurorehabil. Neural Repair 27(1), 45-52 (2013).

24. Kalron, A. \& Achiron, A. Postural control, falls and fear of falling in people with multiple sclerosis without mobility aids. J. Neurol. Sci. 335(1-2), 186-190 (2013).

25. Kalron, A., Allali, G. \& Achiron, A. Neural correlates of gait variability in people with multiple sclerosis with fall history. Eur. J. Neurol. 10, 1-7 (2018).

26. Lalive, P. H., Elsworth-edelsten, C. Gait variability in multiple sclerosis : A better falls predictor than EDSS in patients with low disability. 447-450 (2016).

27. Punt, M., Bruijn, S. M., Wittink, H., Van De Port, I. G. \& Van Dieën, J. H. Do clinical assessments, steady-state or daily-life gait characteristics predict falls in ambulatory chronic stroke survivors?. J. Rehabil. Med. 49(5), 402-409 (2017).

28. Moon, Y., Sung, J. H., An, R., Hernandez, M. E. \& Sosnoff, J. J. Gait variability in people with neurological disorders: A systematic review and meta-analysis. Hum. Mov. Sci. 47, 197-208 (2016).

29. Schniepp, R., Möhwald, K. \& Wuehr, M. Gait ataxia in humans: Vestibular and cerebellar control of dynamic stability. J. Neurol. 264, 87-92 (2017).

30. Schwid, S. R., Goodman, M., McDermott, M. P., Bever, M., Cook, S. D. Functional measures in MS : What is a reliable change? 2001-2003 (2002).

31. Goldman, M. D., Marrie, R. A., Cohen, J. A. Evaluation of the six-minute walk in multiple sclerosis subjects and healthy controls 383-390 (2008).

32. Bischoff, H. A. et al. Identifying a cut-off point for normal mobility: A comparison of the timed 'up and go' test in communitydwelling and institutionalised elderly women. Age Ageing 32(3), 315-320 (2003).

33. Wrisley, D. M., Marchetti, G. F., Kuharsky, D. K., Whitney, S. L. Reliability, internal consistency, and validity of data obtained with the functional gait assessment. 84(10) (2004).

34. Meyer, C. et al. Familiarization with treadmill walking: How much is enough?. Sci. Rep. 9(1), 1-10 (2019).

35. Killeen, T. et al. Increasing cognitive load attenuates right arm swing in healthy human walking. R. Soc. Open Sci. 4(1) (2017)

36. Kalron, A. The Romberg ratio in people with multiple sclerosis. Gait Posture 54, 209-213 (2017).

37. Filli, L. et al. Profiling walking dysfunction in multiple sclerosis: Characterisation, classification and progression over time. Sci. Rep. 8(1), 1-13 (2018).

38. Havrdova, E. et al. Spatial and temporal characteristics of gait as outcome measures in multiple sclerosis (EDSS 0 to 6.5). J. Neuroeng. Rehabil. 12(1), 14 (2015).

39. Socie, M. J., Sosnoff, J. J. Gait Variability and Multiple Sclerosis Vol. 2013 (2013).

40. Givon, U., Zeilig, G. \& Achiron, A. Gait analysis in multiple sclerosis: Characterization of temporal-spatial parameters using GAITRite functional ambulation system. Gait Posture 29(1), 138-142 (2009).

41. Martin, C. L. et al. Gait and balance impairment in early multiple sclerosis in the absence of clinical disability. Mult. Scler. 12(5), 620-628 (2006).

42. Brach, J. S., Studenski, S., Perera, S., Vanswearingen, J. M. \& Newman, A. B. Stance time and step width variability have unique contributing impairments in older persons. Gait Posture 27, 431-439 (2008).

43. Bower, K. et al. Dynamic balance and instrumented gait variables are independent predictors of falls following stroke. J. Neuroeng. Rehabil. 16(1), 1-9 (2019).

44. Snijders, A. H., Van de Warrenburg, B. P., Giladi, N. \& Bloem, B. R. Neurological gait disorders in elderly people: Clinical approach and classification. Lancet Neurol. 6(1), 63-74 (2007).

45. Kasser, S. L., Jacobs, J. V., Foley, J. T., Cardinal, B. J. \& Maddalozzo, G. F. A prospective evaluation of balance, gait, and strength to predict falling in women with multiple sclerosis. YAPMR 92(11), 1840-1846 (2011).

46. Sandroff, B. M., Sosnoff, J. J. \& Motl, R. W. Physical fitness, walking performance, and gait in multiple sclerosis. J. Neurol. Sci. 328(1-2), 70-76 (2013).

47. Su, B. L., Song, R., Guo, L. Y. \& Yen, C. W. Characterizing gait asymmetry via frequency sub-band components of the ground reaction force. Biomed. Signal Process. Control 18, 56-60 (2015).

48. Hsu, A., Tang, P. \& Jan, M. Analysis of impairments influencing gait velocity and asymmetry of hemiplegic patients after mild to moderate. Stroke 9993, 7 (2003).

49. Sen Wei, T., Liu, P. T., Chang, L. W. \& Liu, S. Y. Gait asymmetry, ankle spasticity, and depression as independent predictors of falls in ambulatory stroke patients. PLoS ONE 12(5), 1-14 (2017).

50. Rochester, L., Galna, B., Lord, S. \& Burn, D. The nature of dual-task interference during gait in incident Parkinson's disease. Neuroscience 265, 83-94 (2014). 
51. Selgrade, B. P., Meyer, D., Sosnoff, J. J. \& Franz, J. R. Can optical flow perturbations detect walking balance impairment in people with multiple sclerosis?. PLoS ONE 15(3), 1-16 (2020).

52. Socie, M. J. et al. Footfall placement variability and falls in multiple sclerosis. Ann. Biomed. Eng. 41(8), 1740-1747 (2013).

53. Comber, L., Galvin, R. \& Coote, S. Gait \& Posture Gait de fi cits in people with multiple sclerosis : A systematic review and. Gait Posture 51, 25-35 (2017).

54. Svoboda, Z. et al. Variability of spatial temporal gait parameters and center of pressure displacements during gait in elderly fallers and nonfallers: A 6-month prospective study. PLoS ONE 12(2), 1-11 (2017).

55. Greene, B. R. et al. Quantitative falls risk assessment using the timed up and go test. Biomed. Eng. IEEE Trans. 57(12), 2918-2926 (2010).

56. Hoskovcová, M. et al. Predicting falls in Parkinson disease: What is the value of instrumented testing in off medication state?. PLoS ONE 10(10), 1-13 (2015).

57. Dicharry, J. et al. A three-dimensional kinematic and kinetic comparison of overground and treadmill walking in healthy elderly subjects. Clin. Biomech. 25(5), 444-449 (2010).

58. Parvataneni, K., Ploeg, L., Olney, S. J. \& Brouwer, B. Kinematic, kinetic and metabolic parameters of treadmill versus overground walking in healthy older adults. Clin. Biomech. 24(1), 95-100 (2009).

59. Riley, P. O., Paolini, G., Della Croce, U., Paylo, K. W. \& Kerrigan, D. C. A kinematic and kinetic comparison of overground and treadmill walking in healthy subjects. Gait Posture 26(1), 17-24 (2007).

60. Schellenbach, M., Lövdén, M., Verrel, J., Krüger, A. \& Lindenberger, U. Adult age differences in familiarization to treadmill walking within virtual environments. Gait Posture 31(3), 295-299 (2010).

\section{Author contributions}

A.E. collected and analysed data, prepared the tables, wrote and reviewed the manuscript. L.F. designed the protocol, collected and analysed data, performed statistical analysis, prepared the tables and figures, wrote and reviewed the manuscript. P.H. collected data, prepared figures and reviewed the manuscript. L.W., K.R. reviewed the manuscript. J.A.P., D.S. supervised the study and reviewed the manuscript. A.E. has full access to all the data of the study and takes responsibility for the data integrity and the accuracy of the data analysis. All authors gave final approval for publication.

\section{Competing interests}

The authors declare no competing interests.

\section{Additional information}

Correspondence and requests for materials should be addressed to A.E.

Reprints and permissions information is available at www.nature.com/reprints.

Publisher's note Springer Nature remains neutral with regard to jurisdictional claims in published maps and institutional affiliations.

(c) (i) Open Access This article is licensed under a Creative Commons Attribution 4.0 International cc) License, which permits use, sharing, adaptation, distribution and reproduction in any medium or format, as long as you give appropriate credit to the original author(s) and the source, provide a link to the Creative Commons licence, and indicate if changes were made. The images or other third party material in this article are included in the article's Creative Commons licence, unless indicated otherwise in a credit line to the material. If material is not included in the article's Creative Commons licence and your intended use is not permitted by statutory regulation or exceeds the permitted use, you will need to obtain permission directly from the copyright holder. To view a copy of this licence, visit http://creativecommons.org/licenses/by/4.0/.

(C) The Author(s) 2020 